\title{
New Roles for Community Pharmacists in Modern Health Care Systems: A Challenge for Pharmacy Education and Research
}

\author{
Olivier Bugnon ${ }^{\star a b}$, Denise Hugentobler-Hampaï ${ }^{b}$, Jérôme Berger ${ }^{\mathrm{b}}$, and Marie Paule Schneider ${ }^{\mathrm{b}}$
}

\begin{abstract}
The academic activities led by the Unit of Community Pharmacy can be classified as translational. Our group is interested in person-centered pharmaceutical services aimed at a more responsible use of drugs (effectiveness, safety, efficiency) in collaboration with physicians and other health care professionals in a primary care setting. The following domains of education and research are high priorities for our group: medication therapy management, medication adherence, integrated care, individualization of therapies, care management for the elderly and e-health.
\end{abstract}

Keywords: Community pharmacy services - Interprofessional relations in health care $\cdot$ Medication adherence . Medication therapy management $\cdot$ Pharmaceutical care

\section{Introduction}

The ultimate goal of a drug is to prevent, relieve and/or cure a specific health problem. Although this seems obvious, the factors associated with treatment are complex. For example, medications are among the leading preventable causes of death in many countries, ${ }^{[1]}$ and the median incidence of preventable adverse drug events requiring hospital admission was reported in a systematic review to be 4.5 per 1 '000 person-months. ${ }^{[2]}$ Moreover, adherence to medication among chronic patients averages only $50 \%,{ }^{[3]}$ and the costs of drugs and of their negative outcomes are an increasing concern for health care expenses. The review of the Swiss health care system, which was published in 2011 by the Organisation for Economic Co-operation and Development (OECD) and the World Health Organization (WHO), ${ }^{[4]}$ acknowledged the satisfactory performance of our health care system but also emphasized the need to adapt to future challenges. This review particularly recommended diminish-

${ }^{\star}$ Correspondence: Prof. Dr. O. Bugnon ${ }^{\text {ab }}$

E-mail: olivier.bugnon@hospvd.ch

aSchool of pharmaceutical sciences

Community Pharmacy Unit

University of Geneva, University of Lausanne

${ }^{\mathrm{b} C o m m u n i t y ~ P h a r m a c y}$

Department of Ambulatory Care \& Community

Medicine

University of Lausanne, Lausanne ing hospital services while promoting general health by strengthening public health activities and primary care.

\section{Responsible Use of Medications}

One major trend in pharmacy practice has been the movement away from its original role in medicinal product supply and dispensation and towards a more inclusive focus on personal care. This evolution and the related changes in quality care standards were officially defined in 2011 in the form of a joint publication from the WHO and the International Pharmaceutical Federation (FIP). ${ }^{[5]}$ Collaborative and seamless care is critical for addressing challenges faced by all health care systems, which include a large aging population, limited financial resources (mostly due to increases in care requests), shortages of health care workers, more complex requirements and the information needs of the population and the greater use of e-health technologies. Collaborative pharmacy practice is defined by the FIP as "the clinical practice where pharmacists collaborate with other health care professionals in order to care for patients, caregivers and the public". ${ }^{[6]}$

Efficacy and effectiveness are terms used to describe how well a treatment achieves the desired outcomes. Efficacy describes "how well a therapy can work" under optimal conditions (e.g. randomized clinical studies), whereas effectiveness refers to "how well it does work" in routine care. A third parameter, efficiency, measures the performance of any intervention by defining its ability to produce the desired outcomes with a minimum of ef- fort, expense or waste. Pharmacy schools should produce students with high degrees of competence to bridge the gap between the 3 Es - the efficacy, effectiveness and efficiency - of medical treatments. Indeed, the role of the pharmacist, as well as his/ her compensation, will fluctuate depending on the added value of their services for achieving a more responsible use of medicines.

In this context, the School of Pharmaceutical Sciences (University of Geneva, University of Lausanne) created the unit of Community Pharmacy in 2007 with initial financial help from pharmaSuisse (Swiss Association of Pharmacists). This unit, which is led by Professor Olivier Bugnon, is located in Lausanne at the Department of Ambulatory Care \& Community Medicine (PMU de Lausanne, University of Lausanne, Switzerland) [Box 1]. By establishing collaborations between family physicians, specialists, dentists, nurses and pharmacists, the interdisciplinary academic environment at the PMU presents a unique opportunity for community pharmacy developments. The proximity of the University Hospital (CHUV) also promotes the study of the continuum of care as well as the ability to interface with the hospital pharmacy (including the clinical pharmacy). The present article illustrates the vision and activities of our group, which performs the tasks of a public community pharmacy, a drug information and education center [Box 2] and a research group focused on Community Pharmacy Practice [Box 3].

The required health care system evolution demands a complete culture and workflow management change that should 


\section{Box 1. Facts and Figures of the Pharmacy at the PMU in 2011}

- A total of thirty collaborators were hired in three sectors (public community pharmacy, drug information and education, and research); of these, five collaborators are $\mathrm{PhD}$ students in the pharmaceutical sciences and six are pharmacists in postgraduate training.

- The quality management system in place is in concordance with ISO 9001:2008, Healthmark and ISAS QMS-Pharma 2010 standards.

- Over 23'000 validations of medical prescriptions, 3'500 contacts with travelers, 8'000 contacts for nonprescription drugs, and 3'400 contacts for the supply of drugs in the outpatient care units at the PMU were performed.

- Immunization and counseling services for travelers were performed by two specialized pharmacists in collaboration within the Tropical and Travel Medicine Clinic at the PMU.

- There have been 1'657 motivational interviews conducted with chronic patients and 2'185 pillboxes prepared (medication adherence programs).

- The drug information sector produced over 1'300 clinical and scientific replies to the questions of the physicians and the nurses at the PMU (70\% from the Travel Medicine Clinic at the PMU).

- Nearly 200 hours of undergraduate teaching have been conducted, which served as a major contribution to the final exams for pharmacy students (Masters in Pharmacy and federal exams).

- Keywords characterizing the education and scientific research conducted include the following: community pharmacy services, medication therapy management, medication adherence, collaborative and integrated care, individualization of the therapies, care management for the elderly and e-health.

begin with greater self-confidence on the part of the pharmacist for his/her own professional and interpersonal skills. The implementation of new practices should combine research and development, education and coaching, regulations and quality of care standards, management and business models. Our group contributes to this process by reinforcing collaboration within the University (between the social sciences, medicine and economic sciences in particular) but also assists the practitioners by participating in the undergraduate, postgraduate and continuous education curricula. In particular, our group pursues the creation of long-term partnerships between the University and the practitioners, which should stimulate the development of an innovative research practice.

\section{Physicians-Pharmacists Quality Circles (PPQCs) in the Primary Care Setting and in Nursing Homes}

The PPQCs organize relationships between volunteer family physicians and community pharmacists. Encouraged by the pharmacists, the physicians study how to optimize their prescription profiles, and medication data are issued from the community pharmacy invoices to the health insurance companies. The following factors are key facilitators of the PPQCs: a structured, interdisciplinary continuous quality improvement and education process; local networking; feedback in the form of comparative and detailed data regarding costs, drug choice, and frequency of prescribed

- Communication skills and ethics $(17 \mathrm{~h})$ promotion and prevention $(24 \mathrm{~h})$

- Pharmaceutical triage $(34 \mathrm{~h})$
Adapted from the PPQC workflow in 2002, pharmacists from the canton of Fribourg (Switzerland) have successfully dispensed a remunerated program of pharmaceutical care services (PCS) for residents living in nursing homes $(\mathrm{NH})$. In Switzerland, as in other countries, $\mathrm{NH}$ are becoming more medically oriented and are therefore facing increasing issues with drug safety and drug costs. Our group contributes to the development and implementation of this collaborative program and is responsible for its scientific evaluation (Box 3; ref. [6]). Previous work from our group demonstrated a nine-year sustained cost-containment impact of the PCS and a significant decrease in the mortality rate without any cost transfer towards the hospital. The first step of the current econometric analysis has identified specific variables that illustrate the added value of this advanced collaborative pharmacy service, and further analyses are being performed to elaborate upon a predictive model of drug costs. The collaborative process and its economic and clinical outcomes on medicine use have been hailed by health insurance companies and health care authorities as a positive advance. In reply to a Legislative Council member of the canton of Vaud in 2008, the Health Minister mandated a project to elaborate a model for drug cost containment and patient safety in the NH of Vaud through an analogous collaboration between pharmacists and family physicians. PPQC work process demonstrates sustainable results, such as significant cost savings, greater administration of generics, a reflection on patient safety and the placement of 'new' drugs in patient therapies (Box 3; ref. [3]). The mutual trust gained by the participants of the PPQCs provides a solid basis for implementing more comprehensive collaborative programs, such as medication reviews, adherence-enhancing interventions, or disease management approaches.

\section{Medication Adherence}

Patients with chronic conditions generally demonstrate insufficient adherence to medication schedules and dosing, which has very negative consequences in terms of quality and efficiency of care. ${ }^{[3]}$ This fact is alarming but not surprising, as medication adherence is related to profound individu-

Box 2. Synthesis of the course programs during the $2^{\text {nd }}$ year of the Masters in Pharmacy at the School of Pharmaceutical Sciences (University of Geneva, University of Lausanne)

30 credits (ECTS) for specific pharmacy practice lectures:

- Pharmacotherapy: pharmaceutical validation of medical prescriptions (52 h); medical devices $(12 \mathrm{~h})$, veterinary pharmacy $(8 \mathrm{~h})$

- Complementary medicine and phytotherapy (16 h)

- Health economics, Health care system organization and laws (62 h)

- Public health (34 h): applied epidemiology (6 h); immunizations (4 h); health

- Pharmaceutical care: in primary care (78 h) and in hospital (36 h)

- Formulation and pharmaceutical technology $(68 \mathrm{~h})$

30 credits (ECTS) for a practice training (20 weeks in a community pharmacy and 10 weeks in a community pharmacy or in a hospital pharmacy). 
Box 3. Key publications illustrating the research conducted by the Unit of Community Pharmacy at the School of Pharmaceutical Sciences (University of Geneva, University of Lausanne)

[1] I. Krummenacher, M. Cavassini, O. Bugnon, M.-P. Schneider, 'An interdisciplinary $\mathrm{HIV}$-adherence program combining motivational interviewing and electronic antiretroviral drug monitoring', AIDS Care 2011, 23, 550-561.

[2] I. Krummenacher, M. Cavassini, O. Bugnon, R. Spirig, M.-P. Schneider, The Swiss HIV Cohort Study, 'Antiretroviral adherence program in HIV patients: a feasibility study in the Swiss HIV Cohort Study', Pharm. World Sci. 2010, 32, 776-786.

[3] A. Niquille, M. Ruggli, M. Buchmann, D. Jordan, O. Bugnon, 'The Nine-Year Sustained Cost-Containment Impact of Swiss Pilot Physicians-Pharmacists Quality Circles', Ann. Pharmacotherapy 2010, 44, 650-657.

[4] A. Niquille, O. Bugnon, 'Relationship between drug-related problems and health outcomes: a cross-sectional study among cardiovascular patients', Pharm. World Sci. 2010, 32, 512-519.

[5] J.-F. Locca, C. Bula, S. Zumbach, O. Bugnon, 'Pharmacological treatment of behavioral and psychological symptoms of dementia (BPSD) in nursing homes: development of practice recommendations in a Swiss canton', JAMDA 2008, 9 , 439-448.

[6] J.-F. Locca, M. Ruggli, M. Buchmann, J. Huguenin, O. Bugnon, 'Development of pharmaceutical care services in nursing homes: practice and research in a Swiss canton', Pharm. World Sci. 2008, 31, 165-173.

[7] J.-M. Krähenbuhl, B. Kremer, B. Guignard, O. Bugnon, 'Practical evaluation of the drug-related problem management process in Swiss community pharmacies', Pharm. World Sci. 2008, 30, 777-786.

ality and the numerous representations of any disease. Furthermore, adherence does not declare itself; it is a dynamic behavior over time that will vary depending on events in the life of each individual.

Information technologies and e-Health within this domain can represent a vector for local interdisciplinary collaboration and the individualization of support for medication adherence based on the model of pharmaceutical care programs developed by our group. At the heart of this intervention is motivational interviewing, which is led by the pharmacists and is meant to integrate drug taking into the daily routine of each chronically ill patient. An electronic pillbox provides data that reflect the medication schedule of the patient and evaluate the persistence and execution of medication adherence; these data provide an objective basis of reflection for the barriers and facilitators of adherence. These electronic pillbox reports, as well as the pharmaceutical reports of the elements of the interview, are transmitted to the medical and nurse teams, and these documents promote the continuity of care and consolidate the medical decisions. The results of medication adherence services at the pharmacy of the PMU have demonstrated that this program can provide concrete outcomes. For example, of a group of 102 complex HIV patients, who were followed in a coordinated manner by the service of infectiology at the university hospital (CHUV) and the pharmacy of the PMU over approximately 13 months, the measured persistence was $87 \%$ and the quality of execution was $88 \%$, which are both very high rates. In a sub-group of 45 patients who had no change in their therapy (with the exception of participation in the program), the proportion of those who had undetectable viral loads increased from 38 to $71 \%$. This success was confirmed by the very high retention rate $(92 \%)$ of the program and the high expressed satisfaction rate (Box 3; ref. [1] and [2]). In 2011, over 1'600 pharmacist-led adherence consultations have been performed and 2'100 pillboxes have been prepared at the pharmacy of the PMU in partnership with diverse medical and care teams (e.g. HIV, oncology, diabetes, metabolic syndrome, transplantation, and dialysis) [Box 1].

Our group is also working to develop a web platform dedicated to interdisciplinary person-centered care programs, which will guarantee the traceability of all interventions. These programs share information among the participants and provide remote support to the patients (e.g. sms/ text message reminders) as well as the professionals (e.g. training material). The structured organization of these data via the web makes it easier to scientifically evaluate the performance of this innovative model of integrated care.

\section{Risk Evaluation and Mitigation Strategies}

The dynamism of the pharmaceutical industry leads to the marketing of effective new drugs against severe pathologies (cancers, HIV, hepatitis, graft rejection, multiple sclerosis, etc.), which results in the emergence of new groups of chronically treated patients. These drugs, which are increasingly accessible in oral or autoinjectable forms, will likely offer greater autonomy to patients, provided the patients are able to adopt individualized risk mitigation strategies and receive seamless care at specialized care centers as well as community pharmacies. With this aim, our group currently studies the professional (i.e. education, management, risk management, and person-centered collaborative frameworks), technological (e-health) and organizational (i.e. integrated and seamless care business models) aspects that will enable specialized community pharmacists to contribute to the responsible and integrated use of new drugs.

\section{Medication Review and Medication Therapy Management}

Medication reviews (MRs) for chronic treatments consist of a systematic, pharmacist-led process built on evidence-based practice and aim to detect and solve drugrelated problems (DRPs). Different levels of MRs exist based on the availability of medication data, clinical data and patient interviews. Since 2010, medication use reviews (MURs) have been remunerated by health insurance in Switzerland. The goal of this service is to support medication concordance and patient empowerment, regarding the following aspects: the assessment of actual use, motivation, understanding and individual experience; the identification of DRPs that may disrupt medication adherence; and the identification of problems of drug access.

More advanced clinical MRs are neither routinely delivered nor remunerated in Switzerland. However, our group conducted exploratory studies to assess the feasibility of this service for the community pharmacy and the quality of the pharmaceutical recommendations made to the general practitioners. Our group studies the readiness of patients to accept a MR and also the relationship between the DRPs detected and the economic, clinical and humanistic outcomes. In 2007, a prospective study was performed in the French-speaking region of Switzerland and involved 11 pharmacists and 61 family physicians and a total of 85 patients aged 56 to 75 years who had been treated with at least one cardiovascular drug (Box 3; ref. [4]). The pharmaceutical intervention involved a retrospective analysis (over 6 months) of the medication record as well as information collected during a semistructured interview with the patient and clinical data provided by the family physi- 
cian. The pharmacist then provided recommendations, which the physician was free to choose whether to implement, and the measured results should encourage practitioners to follow the steps recommended. Drug-related problems with clinical or economical consequences were observed among $91 \%$ of the included patients. $70 \%$ of the pharmacists' recommendations were considered a priority by the family physicians. Furthermore, a statistical link was established between the presence of DRPs and the failure to control cardiovascular risk factors, a lower quality of life and higher healthcare costs.

\section{Pharmacy of Addiction}

Switzerland has built its drugs policy on a four-pillar model, consisting of repression, treatment, prevention, and survival aid (in the order of financial investments), to which innovative measures such as heroin prescription treatments are added. Pharmacists have supported this approach and have facilitated accessibility to syringe exchanges, the fabrication and delivery of diverse pharmaceutical formulations of methadone as well as the delivery of prevention messages. In the $21^{\text {st }}$ century, progress in neuroscience, genetics and psychosocial methods for cognitivebehavioral analyses has created a network culture that has enabled collaboration within the entire healthcare workforce. Since 2004, our group has been mandated by the COROMA (Collège Romand de Médecine de l'Addiction) and the Federal Office of Public Health (FOPH) to define and reinforce the contribution of the pharmacist to the interdisciplinary network concerned with the prevention and treatment of addiction.
The studies conducted demonstrated that pharmacists experience the following types of barriers: disparity between the quantity and precision of official documents to be filed in pharmacies; the absence of any document linking the treating physician, the patient and the pharmacist; and the lack of medical and social information. The quality of communication with the physicians was considered adequate, but the communication was often superficial or rare. Although pharmacists are at ease with the fabrication and dispensation of methadone, they have expressed a need for interdisciplinary training in the care of medical-social patients. This need for training on the other forms of addictions, such as those to opioids (e.g. alcohol, cocaine, party drugs, etc.), is even more acute. Our group runs under- and post-graduate training programs, stimulates exchanges between pharmacists within the Frenchspeaking region of Switzerland, and generates procedures of good practice, quality standards and self-assessment tools. The main goal of our work is to promote a communication culture to create partnerships at the local or supra-regional level for the interests of the patients and the society.

\section{Conclusion}

The profession of the pharmacist is changing to adapt to the political and economic evolutions of the health-care system, the needs of the population of the $21^{\text {st }}$ century, newly implemented technological resources and the growing complexity of the responsible use of modern drugs (i.e. safe, effective and economical usage). These new challenges call for further involvement in teaching and research in the schools of pharmacy. Therefore, our
Community Pharmacy Practice group is a pioneer in Switzerland as well as abroad, and our principal strength is to combine a reference center in community pharmacy practice and an university expertise center. It is therefore necessary to emphasize the mutual benefits that can be gained from this proximity to the Faculty of Medicine (via the Department for Ambulatory Care and Community Medicine in Lausanne) and also the benefits of the continuity with the hospital pharmacy. In the future, the goal of our group is to consolidate its clinical and university activities, such that pharmacists will be in a position to contribute to the networks of integrated care and be able to construct a bond of trust with chronically ill patients and the elderly, which is a necessary component for medication efficiency and adherence.

Received: February 27, 2012

[1] WHO, 'The Medicines Safety Team within Quality and Safety of Medicines (QSM)', www. who.int/medicines/areas/quality_safety/safety_ efficacy/en/

[2] L. A. Thomsen, A. G. Winterstein, B Sondergard, L. S. Haugbolle, A. Melander, Ann. Pharmacother. 2007, 9, 1411. Epub July 31, 2007.

[3] WHO, 'Adherence to long-term therapies: evidence for action', 2003.

[4] OECD Reviews of Health Systems SWITZERLAND, 20011, www.oecd.org/ document/48/0,3746, en_2649_37407_485928 16_1_1_1_37407,00.html

[5] Joint FIP/WHO Guidelines on Good Pharmacy Practice (GPP): Standards for quality of pharmacy services; WHO technical report series, No. 961, 45th report of the WHO Expert Committee on specifications for pharmaceutical preparations, WHO, 2011

[6] FIP Statement of Policy: Collaborative Pharmacy Practice. Approved by FIP Council in September, 2010. www.fip.org/statements. 